\title{
FINITELY ADDITIVE GLEASON MEASURES
}

\author{
ANATOLIJ DVUREČENSKIJ
}

(Communicated by Palle E. T. Jorgensen)

\begin{abstract}
We describe the set of all finitely additive measures which attain also infinite values on a quantum logic of a Hilbert space and which are expressible via the generalized Gleason-Lugovaja-Sherstnev formula. We prove that this set consists of those which are regular with respect to the set of all finite-dimensional subspaces. In addition, we show that this regularity does not entail the countable additivity, in general.
\end{abstract}

\section{INTRODUCTION}

One of the basic problems related to the propositional calculus approach to the foundations of quantum mechanics $[2,14]$ is the description of probability measures (called states in physical terminology) on the set of experimentally verifiable propositions regarding a physical system. The set of propositions form an orthomodular partially ordered set, where the order is induced by a relation of implication, and is called a quantum logic. In the more restrictive setting, a quantum logic is assumed to be a complete orthomodular lattice (see [18]).

An important interpretation of a quantum logic is the set $L(H)$ of all closed subspaces of a real or complex Hilbert space $H$, which is an orthomodular complete lattice with respect to the set-theoretical inclusion and the natural orthocomplementation $M \mapsto M^{\perp}=\{x \in H:(x, y)=0$ for each $y \in M\}$. In this interpretation a finite countably additive measure is a map $m: L(H) \longrightarrow[0, \infty)$ satisfying $m\left(\bigvee_{i=1}^{\infty} M_{i}\right)=\sum_{i=1}^{\infty} m\left(M_{i}\right)$ for any sequence $\left\{M_{i}\right\}$ of mutually orthogonal closed subspaces of $H$. The remarkable result of Gleason [9] states that all countably additive measures $m$ on $L(H)$, where $H$ is separable and $\operatorname{dim} H \neq 2$, are in a one-to-one correspondence with positive operators $T$ of the trace class on $H$ via

$$
m(M)=\operatorname{tr}\left(T P^{M}\right), \quad M \in L(H),
$$

where $P^{M}$ denotes the orthoprojector from $H$ onto $M$. This formula has been generalized to nonseparable Hilbert spaces whose dimension is a nonmeasurable

Received by the editors September 7, 1990 and, in revised form, October 2, 1990.

1980 Mathematics Subject Classification (1985 Revision). Primary 81B10, 46C10, 03G12; Secondary 28A60.

Key words and phrases. Hilbert space, quantum logic, measure, Gleason measure, regular measure, bilinear form. 
cardinal by Eilers and Horst [8], Drisch [3], and by Maeda [15] for general $H$ (see also [10]). In view of this Gleason's result, it is natural to look at the more general situation, where the measure is assumed to be only finitely additive and can take infinite values on $L(H)$. Any mapping $m: L(H) \longrightarrow[0, \infty]$ such that

$$
m\left(\bigoplus_{a \in A} M_{a}\right)=\sum_{a \in A} m\left(M_{a}\right)
$$

(here by $\bigoplus_{a \in A} M_{a}$ we denote the join of a system of mutually orthogonal subspaces $\left\{M_{a}: a \in A\right\}$ of $\left.L(H)\right)$, is said to be a finitely additive measure, (countably additive) measure, or completely additive measure, respectively, if (1.2) holds for any finite, countable or arbitrary index set $A$, correspondingly.

Let $\mathbf{n}$ be a cardinal. We say that a finitely additive measure $m$ is $\mathbf{n}$-finite if there is a set $I$ whose cardinal is $\mathbf{n}$ and a set of mutually orthogonal subspaces $\left\{M_{a}: a \in I\right\}$ of $H$ such that $\bigoplus_{a \in A} M_{a}=H$ and $m\left(M_{a}\right)<\infty$ for each $a \in I$. In particular, if $\mathbf{n}=\aleph_{0}$, we say that $m$ is $\sigma$-finite.

The formula (1.1) has been extended to $\sigma$-finite countably additive measures on $L(H)$ by Lugovaja and Sherstnev [13]. To describe their result and the results of the present paper we shall use the following notations. By $\operatorname{Tr}(H)$ we mean the set of all trace operators on $H$. Let $t: \mathscr{D}(t) \times \mathscr{D}(t) \longrightarrow C$ be a (conjugate) bilinear form defined on a linear submanifold $\mathscr{D}(t)$ of $H$ (not necessarily dense or closed in $H$ ), called the domain of $t$. If $t(x, y)=$ $\overline{t(y, x)}$ for all $x, y \in \mathscr{D}(t)$, then $t$ is said to be symmetric; if for a symmetric bilinear form $t$ we have $t(x, x) \geq 0$ for all $x \in \mathscr{D}(t)$, then $t$ is said to be positive. For $M \in L(H), t \circ P^{M}$ will denote the (conjugate) bilinear form with domain $\mathscr{D}\left(t \circ P^{M}\right)=\left\{x \in H: P^{M} x \in \mathscr{D}(t)\right\}$, given by $t \circ P^{M}(x, y)=$ $t\left(P^{M} x, P^{M} y\right), x, y \in \mathscr{D}\left(t \circ P^{M}\right)$. If $t \circ P^{M}$ is induced by an operator $T \in$ $\operatorname{Tr}(H)$, that is, $t \circ P^{M}(x, y)=(T x, y)$ for all $x, y \in H$, then we shall write $t \circ P^{M} \in \operatorname{Tr}(H)$, and we put $\operatorname{tr} t \circ P^{M}=\operatorname{tr} T$. For a nonzero $x$ in $H, P_{x}$ will denote the one-dimensional subspace of $H$ generated by $x$.

Lugovaja and Sherstnev [13] proved that for any $\sigma$-finite measure $m$ on $L(H)$ of a separable Hilbert space $H$, with $m(H)=\infty$, there exists a unique positive bilinear form $t$ defined on a dense domain $\mathscr{D}(t)$ such that

$$
m(M)= \begin{cases}\operatorname{tr} t \circ P^{M} & \text { iff } t \circ P^{M} \in \operatorname{Tr}(H) \\ \infty & \text { otherwise. }\end{cases}
$$

This result has been extended by the author [4] to any $\sigma$-finite $\sigma$-additive measure on $L(H)$ of a Hilbert space $H$ whose dimension is a nonmeasurable cardinal $\neq 2$, and the formula (1.3) will be called the Gleason-LugovajaSherstnev formula, and measures which are expressible via (1.3) are called Gleason measures.

An important notion in measure theory is regularity which in some cases implies the $\sigma$-additivity of finitely additive measures. In the present paper, we shall show that the set of all Gleason measures on $L(H)$ coincides with the set of all $\mathscr{P}(H)_{\infty}$-regular finitely additive measures on $L(H)$ which can take infinite values. Moreover, they are in a one-to-one correspondence with positive bilinear forms with dense domains in $H$. 


\section{REGULAR FINITELY ADDITIVE MEASURES}

A finitely additive measure $m$ on $L(H)$ has the $L-S$ property (L-S stands for Lugovaja - Sherstnev) if (i) there is a two-dimensional subspace $Q$ of $H$ such that $m(Q)<\infty$, and (ii)

$$
D(m)=\left\{x \in H: m\left(P_{x}\right)<\infty\right\} \cup\{0\}
$$

is dense in $H$. According to [13], $D(m)$ is a linear subspace of $H$.

Denote by $\mathscr{P}(H)$ the set of all finite-dimensional subspaces of $H$. A finite, finitely additive measure $m$ on $L(H)$ is said to be $\mathscr{P}(H)$-regular, if for any $M \in L(H)$ and any $\varepsilon>0$ there is a finite-dimensional subspace $N$ of $M$ such that $m\left(M \wedge N^{\perp}\right)<\varepsilon$. In [7], it was shown that a finite finitely additive measure $m$ on $L(H)$, such that $\operatorname{dim} H \neq 2$, is $\mathscr{P}(H)$-regular iff $m$ is of the form (1.1), or, equivalently, iff $m$ is completely additive.

For finitely additive measures on $L(H)$ such that $m(H)=\infty$ the notion of regularity is generalized in the following manner: Let $R_{\infty}=[0, \infty]$. Then $R_{\infty}$ can be considered as a Hausdorff uniform additive semigroup, where the uniformity is determined by a semi-invariant metric $p_{\infty}: R_{\infty} \times R_{\infty} \longrightarrow[0,1]$ such that $p_{\infty}(u, v)=\left|\frac{u}{1+u}-\frac{v}{1+v}\right|, u, v \in R_{\infty}$, with the convention that $\frac{(+\infty)}{1+(\infty)}=$ 1. A finitely additive measure $m$ on $L(H)$ is said to be $\mathscr{P}(H)_{\infty}$-regular if given $M \in L(H)$ and $\varepsilon>0$ there is a finite-dimensional subspace $N$ of $M$ such that $p_{\infty}(m(M), m(N))<\varepsilon$. For finite finitely additive measures both notions of regularity coincide.

Lemma 2.1. Let $H$ be a Hilbert space and $t$ be a positive bilinear form such that $\mathscr{D}(t)$ is dense in $H$. Then the mapping $m_{t}: L(H) \longrightarrow[0, \infty]$ given by

$$
m_{t}(M)= \begin{cases}\operatorname{tr} t \circ P^{M} & \text { iff } t \circ P^{M} \in \operatorname{Tr}(H), \\ \infty & \text { otherwise, }\end{cases}
$$

is a $\mathscr{P}(H)_{\infty}$-regular, finitely additive measure with the $L-S$ property.

Proof. The proof of the finite additivity of $m_{t}$ is similar to the one given in [12] for the case of a separable Hilbert space, and to be selfcontained, we present it here as we shall need it in the second part.

Suppose that $M=\bigoplus_{i=1}^{n} M_{k}, M_{k} \in L(H), k=1, \ldots, n, M_{k} \perp M_{i}$ for $k \neq i$. We claim that

$$
m_{t}(M)=\sum_{k=1}^{n} m_{t}\left(M_{k}\right)
$$

There are three possibilities:

(i) $M \nsubseteq \mathscr{D}(t)$. Then there is an integer $k$ such that $M_{k} \nsubseteq \mathscr{D}(t)$, so that (2.3) holds.

(ii) $M \subseteq \mathscr{D}(t), t \circ P^{M} \notin \operatorname{Tr}(H)$. Then $M_{k} \subseteq \mathscr{D}(t)$ for any $k=1, \ldots, n$, and there exists an integer $k$ such that $t \circ P^{M_{k}} \notin \operatorname{Tr}(H)$. Indeed, in the opposite case we would have that $t \circ P^{M_{k}} \in \operatorname{Tr}(H)$ for any $k=1, \ldots, n$, and for any 
$x \in H,\|x\|=1$,

$$
\begin{aligned}
t \circ P^{M}(x, x) & =t\left(P^{M} x, P^{M} x\right)=\sum_{k, i=1}^{n} t\left(P^{M_{k}} x, P^{M_{i}} x\right) \\
& \leq \sum_{k, i=1}^{n}\left|t\left(P^{M_{k}} x, P^{M_{i}} x\right)\right| \\
& \leq \sum_{k, i=1}^{n}\left(t \circ P^{M_{k}}(x, x) t \circ P^{M_{i}}(x, x)\right)^{1 / 2} \\
& \leq\left|\sum_{k=1}^{n} t \circ P^{M_{k}}(x, x)^{1 / 2}\right|^{2} \leq n \sum_{k=1}^{n} t \circ P^{M_{k}}(x, x) .
\end{aligned}
$$

In addition, if $\left\{x_{i}\right\}$ is an orthonormal basis of $H$, then $\sum_{i} t \circ P^{M}\left(x_{i}, x_{i}\right) \leq$ $n \sum_{k=1}^{n} \operatorname{tr} t \circ P^{M_{k}}$. This entails that $t \circ P^{M}$ is a bounded bilinear form on $H$, so that $t \circ P^{M} \in \operatorname{Tr}(H)$ which contradicts the assumption, and, therefore, (2.3) holds.

(iii) $M \subseteq \mathscr{D}(t), t \circ P^{M} \in \operatorname{Tr}(H)$. Then $M_{k} \subseteq \mathscr{D}(t)$ and $t \circ P^{M_{k}} \in \operatorname{Tr}(H)$ for any $k=1, \ldots, n$. Let $\left\{x_{j}^{k}\right\}_{j}$ be an orthonormal basis of $M_{k}, k=1, \ldots, n$. Then $\bigcup_{k=1}^{n}\left\{x_{j}^{k}\right\}_{j}$ is an orthonormal basis of $M$, and so $m_{t}(M)=\operatorname{tr} t \circ P^{M}=$ $\sum_{k, j} t\left(P^{M} x_{j}^{k}, P^{M} x_{j}^{k}\right)=\sum_{k=1}^{n} \sum_{j} t\left(P^{M_{k}} x_{j}^{k}, P^{M_{k}} x_{j}^{k}\right)=\sum_{k=1}^{n} \operatorname{tr} t \circ P^{M_{k}}=$ $\sum_{k=1}^{n} m_{t}\left(M_{k}\right)$.

We have proved that $m_{t}$ is a finitely additive measure. Since $\mathscr{D}(t)=D\left(m_{t}\right)$, we see that $m_{t}$ has the L-S property.

Now we show that $m_{t}$ is $\mathscr{P}(H)_{\infty}$-regular. Let $M \in L(H)$ and $\varepsilon>0$ be given. As in the first part of the (present) proof, we have three possible cases:

(i) $M \nsubseteq \mathscr{D}(t)$. Then $m_{t}(M)=\infty$, and there is a unit vector $x \in M$ such that $x \notin \mathscr{D}(t)$. Hence, $m_{t}\left(P_{x}\right)=\infty$ and $p_{\infty}\left(m_{t}(M), m_{t}\left(P_{x}\right)\right)<\varepsilon$.

(ii) $M \subseteq \mathscr{D}(t), t \circ P^{M} \notin \operatorname{Tr}(H)$. Then $\mathscr{D}\left(t \circ P^{M}\right)=H$ and, consequently, $\sum_{i} t\left(x_{i}, x_{i}\right)=\infty$ for any orthonormal basis $\left\{x_{i}: i \in I\right\}$ of $M$. Since $u_{n} \rightarrow u$, for $u_{n}, u \in R_{\infty}$, iff $p_{\infty}\left(u_{n}, u\right) \rightarrow 0$, we conclude that $p_{\infty}\left(m_{t}(M), m_{t}\left(\bigoplus_{i=1}^{n} P_{x_{i}}\right)\right)<\varepsilon$ for sufficiently large $n$.

(iii) $M \subseteq \mathscr{D}(t), t \circ P^{M} \in \operatorname{Tr}(H)$. Choose an orthonormal basis $\left\{x_{i}\right\}$ in $M$. Then there exists an integer $n$ such that $p_{\infty}\left(m_{t}(M), m_{t}\left(\bigoplus_{i=1}^{n} P_{x_{i}}\right)\right)<\varepsilon$.

Now we describe the set of all $\mathscr{P}(H)_{\infty}$-regular, finitely additive measures on $L(H)$, when $\operatorname{dim} H \neq 2$.

Theorem 2.2. Let $m$ be a $\mathscr{P}(H)_{\infty}$-regular, finitely additive measure with the $L-S$ property on $L(H), \operatorname{dim} H \neq 2$. Then there exists a unique bilinear form $t$ with dense domain such that (1.3) holds.

Proof. For our $m$, define $D(m)$ via (2.1). Suppose that $m(M)<\infty$. The $\mathscr{P}(H)_{\infty}$-regularity of $m$ entails that the restriction $m \mid L(M)$ is a $\mathscr{P}(M)$-regular, finitely additive measure, and in view of the result in [7], $m \mid L(M)$ is completely additive on $L(M)$. Due to [15] (see also [10]), there exists a positive operator $T_{M}$ of the trace class on $M$ and, consequently, we may assume that $T_{M} \in \operatorname{Tr}(H)$, such that $m(N)=\operatorname{tr}\left(T_{M} P^{N}\right), \quad N \in L(M)$. 
We define a bilinear form $t$ with $\mathscr{D}(t)=D(m)$ putting $t(x, x)=\left(T_{M} x, x\right)$ whenever $x \in M, 3 \leq \operatorname{dim} M<\infty, m(M)<\infty$. Using the Gleason theorem for finite-dimensional subspaces of $S, t$ is a well-defined mapping, because if $x \in M_{1}, x \in M_{2}, 3 \leq \operatorname{dim} M_{1}, \operatorname{dim} M_{2}<\infty, m\left(M_{i}\right)<\infty, i=1,2$, then $m\left(M_{1} \vee M_{2}\right)<\infty$, since $\mathscr{D}(t)$ is a linear submanifold of $S$ containing $M_{1}$ and $M_{2}$, and $t(x, x)=\left(T_{M_{1}} x, x\right)=\left(T_{M_{1} \vee M_{2}} x, x\right)=\left(T_{M_{2}} x, x\right)$.

Define a $\mathscr{P}(H)_{\infty}$-regular, finitely additive measure $m_{t}$ on $L(H)$ via (2.2). We shall show that $m(M)=m_{t}(M)$ for any $M \in L(H)$.

Note that the $\mathscr{P}(H)_{\infty}$-regularity of $m$ entails that for any $M \in L(H)$ there exists an increasing sequence of subspaces of $M,\left\{M_{n}\right\}$, such that

$$
m(M)=\lim _{n} m\left(M_{n}\right) .
$$

Suppose now that $M$ is a finite-dimensional subspace of $H$. If $m(M)=\infty$, then $M \nsubseteq \mathscr{D}(t)$, which yields $m_{t}(M)=\infty$. If $m(M)<\infty$, suppose that $x_{1}, \ldots, x_{n}$ is an orthonormal basis of $M$ and complete it to an orthonormal basis $\left\{x_{i}\right\}$ of $H$. Then

$$
\begin{aligned}
m(M) & =\sum_{i=1}^{n} m\left(P_{x_{i}}\right)=\sum_{i=1}^{n} t\left(x_{i}, x_{i}\right) \\
& =\sum_{i} t \circ P^{M}\left(x_{i}, x_{i}\right)=\operatorname{tr} t \circ P^{M}=m_{t}(M) .
\end{aligned}
$$

Assume that $M$ is an arbitrary subspace of $H$. In view of (2.4), we have

$$
m(M)=\lim _{n} m\left(M_{n}\right)=\lim _{n} m_{t}\left(M_{n}\right) \leq m_{t}(M) .
$$

Hence, $m_{t}(M)=\infty$ if $m(M)=\infty$. If $m(M)<\infty$, then $m \mid L(M)$ is completely additive on $L(M)$. Choosing an orthonormal basis $\left\{x_{i}\right\}$ of $M$, we obtain as above that

$$
m(M)=\sum_{i} m\left(P_{x_{i}}\right)=\sum_{i} t\left(x_{i}, x_{i}\right)=\operatorname{tr} t \circ P^{M}=m_{t}(M) .
$$

This finishes the proof.

\section{Finitely ADDitive MEASURES}

Definition 3.1. A finitely additive measure $m$ on $L(H)$ with the L-S property is said to be a (finitely additive) Gleason measure if $m$ is implemented by a positive bilinear form $t$ via (1.3) such that $\mathscr{D}(t)=D(m)$

Remark 3.2. As a consequence of Theorem 2.2 and Lemma 2.1, the set of all finitely additive Gleason measures coincides with the set of all $\mathscr{P}(H)_{\infty}$-regular finitely additive measures with the L-S property on $L(H)$, if $\operatorname{dim} H \neq 2$.

Remark 3.3. Any $\sigma$-finite or $\mathbf{n}$-finite, finitely additive measure has the L-S property, but the converse is not true, in general, even if $m$ is a Gleason measure as we can see in the following example.

Example 3.4. There exists a $\mathscr{P}(H)_{\infty}$-regular, finitely additive measure $m$ with the L-S property which is not $\mathbf{n}$-finite for any cardinal $\mathbf{n}$. 
Proof. Let $\mathbf{m}$ be an infinite cardinal such that $\mathbf{m}^{\aleph_{0}}>\mathbf{m}$. Then in any Hilbert space of dimension $\mathbf{m}^{\aleph_{0}}$, there exists [5] a submanifold $D$ dense in $H$ containing no orthonormal basis of $H$. Define a bilinear form $t$ with $\mathscr{D}(t)=D$ via $t(x, y)=(x, y)$ for all $x, y \in D$. Then $m_{t}$, defined by (2.2) is the required measure. (For more details, see [5].)

Remark 3.5. Following [12], we note that we can find a bilinear form $t$ with dense domain which produces different $\sigma$-finite finitely additive measures (at least one of them is not $\mathscr{P}(H)_{\infty}$-regular). Indeed, let $\operatorname{dim} H=\aleph_{0}$, and let $M$ be an infinite-dimensional subspace of $H$ such that $t \circ P^{M} \in \operatorname{Tr}(H)$. Then $m_{t}$ defined by (2.2) and $n_{t}$ defined via

$$
n_{t}(M)= \begin{cases}\operatorname{tr} t \circ P^{M} & \text { iff } M \subseteq \mathscr{D}(t), \operatorname{dim} M<\infty, \\ \infty & \text { otherwise }\end{cases}
$$

are those. Moreover, $n_{t}$ has the L-S property.

Note also that using the first part of the proof of Theorem 2.2, we may prove (see also [12]) that for any finitely additive measure $m$ having the L-S property on $L(H), \operatorname{dim} H \neq 2$, there exists a unique bilinear form $t$ with dense domain such that

$$
m(M)=\operatorname{tr} t \circ P^{M} \quad \text { if } \quad \operatorname{dim} H<\infty, M \subseteq \mathscr{D}(t) .
$$

As it has been noted, any finite, $\mathscr{P}(H)$-regular, finitely additive measure is necessarily completely additive, and, therefore, $\sigma$-additive. For measures with infinite values we posed in [6] an open problem whether a $\sigma$-finite, $\mathscr{P}(H)_{\infty}$ regular, finitely additive measure on $L(H)$ is countably additive. We show that the answer is negative, in general. The following example is from [17]: Let $\left\{x_{i}: i \in I_{0}\right\}$ be an orthonormal basis of a separable, infinite-dimensional Hilbert space $H$, which is a part of a Hamel basis $\left\{x_{i}: i \in I\right\}$. Fix an element $x_{i_{0}} \in\left\{x_{i}: i \in I \backslash I_{0}\right\}$ and define a bilinear form $t$ with $\mathscr{D}(t)=H$ via

$$
t\left(\sum_{i \in A} \alpha_{i} x_{i}, \sum_{i \in A} \alpha_{i} x_{i}\right)=\left|\alpha_{i_{0}}\right|^{2},
$$

where $A$ is an arbitrary finite subset of $I$ containing $i_{0}$. Then $m_{t}$, defined by (2.2), is a $\sigma$-finite, $\mathscr{P}(H)_{\infty}$-regular, finitely additive measure which is not $\sigma$-additive (since $t\left(x_{i}, x_{i}\right)=0$ for any $\left.i \in I_{0}\right)$.

The former example is a particular case of a so-called singular bilinear form. We recall that according to Simon [17], for any positive bilinear form $t$ there exists a unique pair of positive bilinear forms $t_{r}$ and $t_{s}$ such that

$$
t=t_{r}+t_{s}, \quad \mathscr{D}(t)=\mathscr{D}\left(t_{r}\right)=\mathscr{D}\left(t_{s}\right),
$$

where $t_{r}$ is the largest closable positive bilinear form dominated by $t$ (and $\left.t_{s}=t-t_{r}\right)$. If $t_{r}=0, t$ is named singular.

In view of [11] and [5], any measure $m_{t}$ on $L(H)$ defined by (2.2) is only a $\mathscr{P}(H)_{\infty}$-regular, finitely additive measure which is not countably additive whenever $t$ is singular. Moreover, $m_{t}$ defined via (2.2) is $\sigma$-additive iff

$$
\left(\overline{t \circ P^{M}}\right)_{r} \in \operatorname{Tr}(H) \text { implies } t \circ P^{M} \in \operatorname{Tr}(H),
$$

where $\left(\overline{t \circ P^{M}}\right)_{r}$ is the regular part of the closure $\overline{t \circ P^{M}}$ of $t \circ P^{M}$ (see [11, 5]). 
We want to mention an interesting result of Lugovaja [12], who proved that for any $\sigma$-finite, finitely additive measure $m$ on $L(H), \operatorname{dim} H=\aleph_{0}$, there exists the largest $\sigma$-finite, completely additive measure $m_{r}$ which is majorized by $m$. This $m_{r}$ is determined by the closure $\overline{t_{r}}$ of $t_{r}$ from the Simon decomposition (3.2) via (2.2). But, in general, $m$ is not decomposable as $m=m_{r}+m_{s}$ with $m_{s}$ vanishing on all finite-dimensional subspaces, which is true for finite finitely additive measure [1, Proposition 2].

We note that another type of regularity which implies the countable additivity is discussed in [16].

\section{OPEN PROBLEM}

Let $m$ be a finitely additive measure on $L(H)$. An element $M_{0}$ of $L(H)$ is said to be a support of $m$ if $m(N)=0$ precisely when $N \perp M_{0}$. It is clear that if $m$ has a support, then it is unique. In [6], we posed an open problem whether a finite, finitely additive measure having a support is necessarily countably additive (and, consequently, completely additive). We give a partial answer to this problem.

Proposition 4.1. Any finite, finitely additive measure $m$ on $L(H), \operatorname{dim} H \neq 2$, having a finite-dimensional support is completely additive.

Proof. According to the result of Aarnes [1, Proposition 2], any finite, finitely additive measure $m$ on $L(H), \operatorname{dim} H \neq 2$, is uniquely decomposable as the sum $m=m_{1}+m_{2}$, where $m_{1}$ is a completely additive measure and $m_{2}$ is a finitely additive measure which vanishes on all finite-dimensional subspaces of $H$.

Let $M_{0}$ be a finite-dimensional support of $m$. Then $m_{2}\left(M_{0}\right)=0$ as well as $m_{2}\left(M_{0}^{\perp}\right)=m\left(M_{0}^{\perp}\right)=0$. Therefore, $m_{2}=0$ and $m=m_{1}$, which proves the assertion.

We note that for a finite, finitely additive measure having an infinite-dimensional support, the assertion of Proposition 4.1 is not true, in general, see [6].

Problem. When a finitely additive measure with the L-S property on $\mathrm{L}(\mathrm{H})$, $\operatorname{dim} H \neq 2$, is necessarily completely additive ?

\section{ACKNOWLEDGMENT}

The author is very indebted to the referee for his valuable suggestions and comments which enabled to improve the paper.

\section{REFERENCES}

1. J. F. Aarnes, Quasi-states on $C^{*}$-algebras, Trans. Amer. Math. Soc. 149 (1970), 601-625.

2. G. Birkhoff and J. von Neumann, The logic of quantum mechanics, Ann. of Math. 37 (1936), 823-843.

3. T. Drisch, Generalization of Gleason theorem, Internat. J. Theoret. Phys. 18 (1979), 239243.

4. A. Dvurečenskij, Generalization of Maeda's theorem, Internat. J. Theoret. Phys. 25 (1986), 1117-1124.

5. __ Note on a construction of unbounded measures on a nonseparable Hilbert space quantum logic, Ann. Inst. H. Poincaré 48 (1988), 297-310. 
6. _ Solution to a regularity problem, Proc. Sec. Winter Sch. Meas. Theory, Liptovský Ján (January 7-12, 1990), 1990, pp. 224-227.

7. A. Dvurečenskij, T. Neubrunn and S. Pulmannová, Regular states and countable additivity on quantum logics, Proc. Amer. Math. Soc., 114 (1992), 931-938.

8. M. Eilers and E. Horst, The theorem of Gleason for non-separable Hilbert space, Internat. J. Theoret. Phys. 13 (1975), 419-424.

9. A. M. Gleason, Measures on closed subspaces of a Hilbert space, J. Math. Mech. 6 (1957), 885-893.

10. G. Kalmbach, Orthomodular Lattices, Academic Press, London, 1983.

11. G. D. Lugovaja, Bilinear forms generating measures on projectors, Izv. Vysš. Učebn. Zaved. Matematika 2 (1983), 88.

12. $ـ$ On a construction of unbounded measures on projectors of a Hilbert space, Issled. po priklad. matem. Izd. Kazan Univ. 1984 (1984), 202-205.

13. G. D. Lugovaja and A. N. Sherstnev, On the Gleason theorem for unbounded measures, Izv. Vysš. Učebn. Zaved. Matematika 12 (1980), 30-32.

14. G. Mackey, The mathematical foundations of quantum mechanics, Benjamin, New York, 1963.

15. S. Maeda, Lattice theory and quantum logics, Maki-Shoten, Tokyo, 1980.

16. P. Morales, New results in non-commutative measure theory, Proc. Sec. Winter Sch. Meas. Theory, Liptovský Ján (January 7-12, 1990), 1990, pp. 156-162.

17. B. Simon, A canonical decomposition for quadratic forms with application to monotone convergence theorems, J. Funct. Anal. 28 (1978), 377-385.

18. V. S. Varadarajan, Geometry of quantum theory, Van Nostrand, Princeton, NJ, 1968.

Mathematical Institute, Slovak Academy of Sciences, Štefánikova 49, CS-814 73 Bratislava, Czechoslovakia

Current address: Institute for Theoretical Physics, University of Cologne, Lülpicherstr. 77, D-5000 Cologne, Germany 